\title{
Reliable Relay Node Placement in Large Scale Dense \& Non-Uniform Wireless Sensor Network
}

\author{
Vivekanand Jha \\ Department of Computer Science \\ Indira Gandhi Institute of technology \\ GGSIPU
}

\author{
Neeharika Taneja \\ Department of Computer Science \\ Indira Gandhi Institute of technology \\ GGSIPU
}

\author{
Harshpreet Kaur \\ Department of Computer Science \\ Indira Gandhi Institute of technology \\ GGSIPU
}

\begin{abstract}
A Wireless sensor network(WSN) is composed of a large number of sensor nodes, which are densely deployed either inside the phenomenon or very close to it. Unlike traditional networks, a WSN has its own design and resource constraints. As sensor nodes operate on limited battery power, energy usage is a very important concern in a WSN; and there has been significant research focus that revolves around harvesting and minimizing energy. In the proposed work, assuming the WSN to be large scale and non uniform an algorithm is proposed which includes- selection of cluster head from dominating set node,creation of clusters with one and only one cluster head and Relay node deployment in clusters and between cluster heads. In this algorithm, approach of clustering and relay node placement are combined for efficient utilization of energy, thus maximizing network lifetime.
\end{abstract}

\section{General Terms:}

Clustering, Energy Efficiency, Relay Nodes

\section{Keywords:}

Wireless Sensor Networks, Dominating Set, Cluster Head, Relay Nodes

\section{INTRODUCTION}

A wireless sensor network is composed of hundreds or even thousands of sensor nodes which use wireless links to perform distributed sensing tasks. Each sensor node includes a sensing module, a computing module, memory and a wireless communication module with a very limited communication range. Wireless sensor network has received intensive research attentions due to its enormous application potential in battlefield surveillance, environmental monitoring, biomedical observation and other fields[1]. The three basic requirements for designing efficient wireless sensor networks are scalability, fault-tolerance and energy efficiency. A sensor network, comprising of a number of sensor nodes, is usually required to cover a large geographic area. New sensor nodes may be added to the network and existing sensor nodes may become inoperative at any time. This large scale and frequently changing network requires scalable protocols and algorithms. Factors, such as energy depletion, harsh environmental conditions, and/or malicious attacks may result in node failures in a wireless sensor network. Therefore, survivability of sensor networks is a critical design goal. Moreover, energy is one of the most precious resource in wireless sensor networks. Sensor nodes are normally powered by batteries and can only last for a fairly short period of time if operated at high transmission power levels.

The fact that the energy requirement for transmission is a superlinear function of the transmission distance [1] necessitates short range communication to improve network lifetime [3]. To prolong network lifetime while meeting certain network specifications, one proposed direction is to deploy a small number of relay nodes (RNs) in the WSN such that they can communicate with the SNs and other RNs[3], [4], [5], [6], [7],[8], [9], [13], [15], This is studied under the theme of relay node placement. This problem has received considerable attention from the networking community, with related papers published in MobiCom [11], MobiHoc [5], [12], and Infocom [10], [14], [15]. We follow clustered based relay node placement. Clustering involves grouping of sensors into a group termed as cluster with the cluster head aggregating the data and transmitting it to the sink. Clustering has been adopted by the research community since long to achieve scalability and high energy efficiency and prolong network lifetime in large scale WSN environments. It also stabilizes the network topology. Construction of a stable backbone structure offers better support for efficient communication. The backbone is a subset of sensors that perform data communications and serves the nodes that are not a part of the backbone. Keeping a battery operated sensor node active all the time is not practicable because it depletes the energy resources quickly. The life time of a sensor node can be increased if it can turn on its circuitry for performing the functions only during the specified time slot allotted to it. The sensor node must turn off its circuitry to conserve energy when its time slot has passed [41]. The motivation for incorporating the backbone structure to the sensor network is to partially turn off the nodes which are not part of the backbone. This saves power and prolongs the network lifetime of WSNs. A Dominating Set, DS is defined as a set of nodes in the network such that all nodes in the network which are not in the DS are adjacent to at least one node in the DS. The efficiency of multi-cast or broadcast routing can be improved through the use of a CDS as backbone. A CDS- backbone eliminates redundant broadcasts and saves power. Thus, cluster formation is based on construction of dominating set. 


\section{BACKGROUND WORK}

\subsection{Dominating Set}

A Dominating Set, DS is defined as a set of nodes in the network such that all nodes in the network which are not in the DS are adjacent to at least one node in the DS. When the graph induced by a DS is connected, then it is called CDS. For any arbitrary network, the problem of computing a CDS of minimum cardinality is an NPcomplete problem [41]. The efficiency of multi-cast or broadcast routing can be improved through the use of a DS as backbone. A DS- backbone eliminates redundant broadcasts and saves power. One such approach is

2.1.1 Distributed Single-Phase algorithm for constructing a connected dominating set( DSP-CDS). DSP-CDS is a distributed algorithm. It is designed to achieve three goals. First, it generates a Connected Dominating Set(CDS) fast in a single phase. Secondly, it generates the CDS of competitively small size compared with other distributed algorithms. Thirdly, it efficiently maintains the CDS under network topology changes. In the DSP-CDS algorithm, each node in the network has a unique ID, called nid. A node can be in one of the three states: white, gray, and black. A dominator is in the black state.

A node adjacent to a dominator, if it is not a dominator itself, is in the gray state. All the other nodes (neither black nor gray) are white. A piece is a connected sub-network during the CDS construction. Connected black nodes and their gray neighbours form one piece. A white node itself is a piece. A piece has a unique ID, called pid, known to all nodes in the piece. A piece has a special node called master. The master of a trivial piece is the white node itself, and the master of a non-trivial piece is one of the black nodes. The piece master decides the pid of the piece. The strength of a node indicates the ability of the node to connect with different pieces. The execution of each node is divided into rounds. In each round, a node exchanges status messages with its neighbours, updates its strength, and decides if it should become a dominator. If the new strength is 0 , the node will not change its state (white, gray, or black). If the new strength is greater than 0 , the node decides its new state based on its current status (including the value of strength) and the local knowledge about its neighbours. A node adjacent to a dominator, if it is not a dominator itself, is in the gray state. All the other nodes (neither black nor gray) are white. A piece is connected sub-network during the CDS construction. Connected black nodes and their gray neighbours form one piece. A white node itself is a piece. A piece has a unique ID, called pid, known to all nodes in the piece. A piece has a special node called master.The master of a trivial piece is the white node itself, and the master of a non-trivial piece is one of the black nodes. The piece master decides the pid of the piece. The strength of a node indicates the ability of the node to connect with different pieces. The execution of each node is divided into rounds. In each round, a node exchanges status messages with its neighbours, updates its strength, and decides if it should become a dominator. If the new strength is 0 , the node will not change its state (white, gray, or black). If the new strength is greater than 0 , the node decides its new state based on its current status (including the value of strength) and the local knowledge about its neighbours.

In the DSP-CDS implementation, the strength of a node $\mathrm{i}$ is computed using the following rules:

(1) Rule-1: If node $\mathrm{i}$ is black, its strength is 0 ;
(2) Rule-2: If node i is gray or white, its strength is the sum of the points contributed by its neighbours;

(3) Rule-3: A black or white neighbour contributes 2 points, and a gray neighbour contributes 1 point, with the following exceptions:

-A neighbour with the same pid as node i contributes 0 point to the strength of node $\mathrm{i}$;

-Among the neighbours sharing the same pid only the one that can contribute the greatest points contributes to the strength of node $i$.

\subsection{Clustering}

In most wireless sensor network (WSN) applications nowadays the entire network must have the ability to operate unattended in harsh environments in which pure human access and monitoring cannot be easily scheduled or effeciently managed or its even not feasible at all [46]. Sensors are energy constrained and their batteries usually cannot be recharged. Neighboring sensor nodes generally have the data of similar events because they collect events within a specifc area. If each node individually transmits the collected data to the sink node, a lot of energy will be wasted to transmit similar data to the sink node. The sensor nodes are organized into a number of clusters in order to avoid such energy wastes. Clusterbased architectures improve the resource allocation and reduce the energy consumption, thus prolong the network lifetime as much as possible [30]. Each cluster is monitored and controlled by a node, called Cluster-Head $(\mathrm{CH})$. These cluster heads communicate directly with the base station (BS). Other nodes send the data, sensed from the environment to these $\mathrm{CHs}$. $\mathrm{CHs}$ first aggregate the data from the multiple sensor nodes, and then nally send it directly to the BS. The one algorithm used is LEACH

\subsubsection{Low Energy Adaptive Clustering Hierarchy} LEACH[34] is perhaps the first cluster based routing protocol for WSN, which use a stochastic model for cluster head selection, and has motivated the design of many protocols. Its an hierarchical, probabilistic, distributed, one-hop protocol, with main objectives (a) to improve the lifetime of WSNs by trying to evenly distribute the energy consumption among all the nodes of the network and (b) to reduce the energy consumption in the network nodes (by performing data aggregation and thus reducing the number of communication messages). It forms clusters based on the received signal strength and also uses the $\mathrm{CH}$ nodes as routers to the BS. All the data processing such as data fusion and aggregation are local to the cluster. LEACH forms clusters by using a distributed algorithm, where nodes make autonomous decisions without any centralized control. All nodes have a chance to become $\mathrm{CHs}$ to balance the energy spent per round by each sensor node. Initially a node decides to be a $\mathrm{CH}$ with a probability $\mathrm{p}$ and broadcasts its decision. Specifically, after its election, each $\mathrm{CH}$ broadcasts an advertisement message to the other nodes and each one of the other (non- $\mathrm{CH}$ ) nodes determines a cluster to belong to, by choosing the $\mathrm{CH}$ that can be reached using the least communication energy (based on the signal strength of each $\mathrm{CH}$ message). The role of being a $\mathrm{CH}$ is rotated periodically among the nodes of the cluster to balance the load. The rotation is performed by getting each node to choose a random number $\mathrm{T}$ between 0 and 1 . A node becomes a $\mathrm{CH}$ for the current rotation round if the number is less than the following threshold:

$$
T(i)=\frac{p}{1-p * \operatorname{rmod} \frac{1}{p}}
$$


else $\mathrm{T}(\mathrm{i})=0$ where

$\mathrm{p}$ is the desired percentage of $\mathrm{CH}$ nodes in the sensor population $r$ is the current round number

$\mathrm{G}$ is the set of nodes that have not been $\mathrm{CHs}$ in the last 1/p rounds .

The clusters are formed dynamically in each round and the time to perform the rounds are also selected randomly. Generally, LEACH can provide a quite uniform load distribution in one-hop sensor networks. Moreover, it provides a good balancing of energy consumption by random rotation of CHs. Furthermore, the localized coordination scheme used in LEACH provides better scalability for cluster formation, whereas the better load balancing enhances the network lifetime. However, despite the generally good performance, LEACH has also some clear drawbacks. Because the decision on $\mathrm{CH}$ election and rotation is probabilistic, there is still a good chance that a node with very low energy gets selected as a $\mathrm{CH}$. Due to the same reason, it is possible that the elected $\mathrm{CHs}$ will be concentrated in one part of the network (good CHs distribution cannot be guaranteed) and some nodes will not have any $\mathrm{CH}$ in their range. Also, the $\mathrm{CHs}$ are assumed to have a long communication range so that the data can reach the BS directly. This is not always a realistic assumption because the $\mathrm{CHs}$ are usually regular sensors and the BS is often not directly reachable to all nodes. Moreover, LEACH forms in general one-hop intracluster and intercluster topology where each node should transmit directly to the $\mathrm{CHs}$ and thereafter to the BS, thus normally it cannot be used effectively on networks deployed in large regions.

\subsection{Relay Nodes}

In general, the relay node placement problem has been studied under two perspectives, namely the routing structure and the connectivity requirements. The study based on the routing structure may be further classified into either single tiered or two-tiered [6], [7], [9], [11]. The study based on connectivity can be classified into either connected or survivable [4], [7], [15], [14]. In single-tiered relay node placement, the SNs may also forward packets. In twotiered relay node placement, the SNs transmit their sensed data to an RN or a BS, but do not forward packets for other nodes. In connected relay node placement, the placement of RNs ensures connectivity between the SNs and the BSs. In survivable relay node placement, the placement of RNs ensures biconnectivity between the SNs and the BSs.

Two optimization problems for relay node placement in large scale sensor networks[2], one is called Connected Relay Node Single Cover (CRNSC) problem and another is 2-Connected Relay Node Double Cover (2CRNDC) problem. Two polynomial time approximation algorithms are presented to solve the CRNSC problem. We show that the size of the CRNSC given by the first approximation algorithm is bounded by eight times that of the optimal solution and the second one achieves a performance ratio of 4.5. Moreover, we propose two approximation algorithms to solve the 2CRNDC problem and we also prove that performance ratios associated with them are 6 and 4.5 , respectively.

In a large scale WSN where nodes are non-uniformly distributed we aim to place relay nodes such that relay node is reachable by at least 1 sensor node. We assume the field to have set $\mathrm{S}$, as sensor node such that they have a transmission range of $r$. The sensor nodes are mobile and change topology after some time. Being non-uniformly placed there might be dense areas where more than 20 sensor nodes may be present in 1 metric cube. However, no interference of data while aggregation at relay node is assumed. Relay nodes are used only for receiving and transmitting data bidirectional between sensor nodes and relay nodes or relay nodes and relay nodes or sink and relay nodes. The transmission radius of relay node is $r$ such that $r$ is not same as $R$.

To do this we place Relay nodes hierarchically. Clusters are formed for set $\mathrm{S}$, such that no sensor node is shared by any two clusters and each sensor node in set $S$ is present in at least and at most 1 cluster. Cluster head is chosen based on dominating set nodes from set, $\mathrm{S}$. Dominating set is formed using the following parameters:

\section{-Residual Energy of Nodes}

-Neighbours of Node

Relay node is placed inside clusters as well as outside. Certain constraints such that, a sensor node must have at least 1 relay node to transmit data to it. Per sensor node 1 fault of relay node is tolerated. This provides fault tolerance in relay node placement. Additional relay nodes are placed around cluster head and in between cluster head. The cluster head of each cluster know distance between itself and all the cluster heads in the field and also the distance from the sink. We will also place additional relay nodes around dense areas.

\section{PROPOSED WORK}

In a large scale WSN where nodes are non-uniformly distributed we aim to place relay nodes such that relay node is reachable by at least 1 sensor node. We assume the field to have set $S$, as sensor node such that they have a transmission range of $r$. The sensor nodes are mobile and change topology after some time. Being non-uniformly placed there might be dense areas where more than 20 sensor nodes may be present in 1 metric cube. However, no interference of data while aggregation at relay node is assumed. Relay nodes are used only for receiving and transmitting data bidirectional between sensor nodes and relay nodes or relay nodes and relay nodes or sink and relay nodes. The transmission radius of relay node is $r$ such that $r$ is not same as $R$.

To do this we place Relay nodes hierarchically. Clusters are formed for set $\mathrm{S}$, such that no sensor node is shared by any two clusters and each sensor node in set $S$ is present in at least and at most 1 cluster. Cluster head is chosen based on dominating set nodes from set, S. Dominating set is formed using the following parameters:

\section{—Residual Energy of Nodes}

-Neighbours of Node

Relay node is placed inside clusters as well as outside. Certain constraints such that, a sensor node must have at least 1 relay node to transmit data to it. Per sensor node 1 fault of relay node is tolerated. This provides fault tolerance in relay node placement. Additional relay nodes are placed around cluster head and in between cluster head. The cluster head of each cluster know distance between itself and all the cluster heads in the field and also the distance from the sink. We will also place additional relay nodes around dense areas. In this section we propose the algorithms we use to place relay nodes. We first create a dominating set, followed by clustering and finally relay node placement.

\subsection{Design Parameters}

The algorithm uses some parameters which are discussed below: 
3.1.1 Node Degree. Each node computes its degree. The degree of a node $\mathrm{y}$ is the total number of nodes within the transmission range of $v$.

$$
D_{g}=\sum_{u \in V, u \neq v} D_{u v}
$$

where Duv is the distance between node $\mathrm{u}$ and $\mathrm{v}$.

3.1.2 Transmission range. The transmission range, $\mathrm{Tr}$, of a node is a factor that determines the quality of the clusterhead. This parameter is more relevant in the case of heterogeneous networks.

3.1.3 Transmission range. The transmission range, $\mathrm{Tr}$, of a node is a factor that determines the quality of the clusterhead. This parameter is more relevant in the case of heterogeneous networks.

3.1.4 Fault Tolerance. Some sensor nodes may fail or be blocked due to lack of power, have physical damage or environmental interference. The failure of sensor nodes should not affect the overall task of the sensor network. This is the reliability or fault tolerance issue. Fault tolerance is the ability to sustain sensor network functionalities without any interruption due to sensor node failures .

$$
R(t)=\exp (-\mu t)
$$

\subsection{Proposed Solution}

3.2.1 Construction of Dominating Set. The algorithm used for constructing dominating set is the modified version of DSP-CDS as described in section 2.3

\section{Algorithm 1: DSP-DS}

(1) compute strength ()

// Compute the strength of a node based on the local knowledge about neighbors

compute strength \{

if (no neighbor)

// strength for an isolated node

return InvalidStrength;

new strength $=0$;

if

// Compute the strength using information of neighbors

the node is not black

if (state != black)

\{

// Count points of black or white nodes in the first round for each black/white neighbor i

\{

new strength $+=2+\mathrm{TR} / \mathrm{MD}$;

\}

// Count points of gray nodes in the second round

for each gray neighbor node $\mathrm{i}$

\{

new strength $+=1+\mathrm{TR} / \mathrm{MD}$;

\}
\}

return new strength;

\}

Where $\mathrm{TR}=$ transmission radius

$\mathrm{MD}=$ Maximum distance of that node with any other node

(2) Each node execution program

define InvalidStrength $=1$;

state $=$ white;

// Initial strength value

strength = InvalidStrength;

reset timer $\mathrm{t} 1 \mathrm{=} \mathrm{T} 1$;

reset timer $\mathrm{t} 2=\mathrm{T} 2$;

upon expiration of $\mathrm{t} 1$

\{

// Compute a new strength

$\mathrm{s}=$ compute strength();

broadcast STATUS(nid, state, $s$ );

reset timer $\mathrm{t} 1=\mathrm{T} 1$;

\}

upon expiration of $\mathrm{t} 2\{$

if $($ state $==$ black - strength $==0$ )

return;

// Compute a new strength

strength $=$ compute strength () ;

// Get the maximum strength among neighbors

$(\mathrm{s}, \mathrm{n})=$ get max neighbor () ;

if ((strength, nid) $i(\mathrm{~s}, \mathrm{n}))$

state $=$ black

reset timer $\mathrm{t} 2=\mathrm{T} 2$;

\}

upon receiving a STATUS message $\mathrm{S}\{$

save neighbour $($ S.nid $)=($ S.state, S.strength $)$;

reset timer neighbourTimer(sender.nid) $=$ NeighbourExp;

if $(($ S.state $==$ black $)$ and $($ state $==$ white $))\{$

state=gray;

\}

\{

upon expiration of neighbor Timer(nid) \{

delete neighbor(nid);

\} 
3.2.2 Choose Cluster head. Four factors are being considered while electing cluster head

(1) Percentage of cluster heads required

(2) Residual Energy of a node

(3) Fault tolerance

(4) Degree of node

\section{Algorithm 2: Cluster Head Nomination}

(1) A node $\mathrm{n}$ chooses a random number between 0 and 1 .

(2) If the number is less than a threshold $T(n)$, the node becomes a cluster head for the current round.

(3) The threshhold is set as $t(n)$, where

$$
\begin{gathered}
T(n)=\frac{p}{1-p *\left(\operatorname{rmod} \frac{1}{p}\right)} * \frac{E_{\text {res }}}{E_{\text {in }}} * K_{\text {opt }} * R(t) * D(n) \\
K_{\text {opt }}=\frac{\sqrt{N}}{\sqrt{2 \pi}} * \frac{E_{f s}}{E_{m p}} * \frac{M}{d B S^{2}} \\
R(t)=\exp (-\mu t)
\end{gathered}
$$

(4) where

$$
\mu
$$

$=$ failure rate of sensor node

(5) $\mathrm{D}(\mathrm{n})=$ probability of a node to have maximum degree

(6) Announce $\mathrm{CH}$ status

(7) $\mathrm{CH}$ sends packets to its 1 hop neighbors informing them about its nomination as a cluster head.

(8) Wait for Join request message

(9) $\mathrm{CH}$ waits for the join request message from its neighborhood sensor nodes.

3.2.3 Cluster Formation. Following is the algorithm of the cluster formation :

\section{Algorithm 3 : Cluster Formation}

A sensor node receives the JOIN request messages from more than 1 cluster head.

The decision of which cluster head should it join is dependent on four factors:-

(1) Residual energy of a cluster head

(2) Distance between $\mathrm{CH}$ and node

(3) Distance between $\mathrm{CH}$ and base station

(4) RSSI- Received Signal Strength Indicator

A node sends the join message to the selected $\mathrm{CH}$

3.2.4 Relay Node Placement. It involves 2 algorithms. These can be described as :
4.2.4.1 WITHIN CLUSTER: Ensure connected relay node single cover(CRNC)

Given a set of locations of non-uniformly distributed sensor nodes $\mathrm{S}$, the communication range of sensor nodes , SN Range, and the communication range of relay nodes RN Range, find the minimum number of relay nodes and their corresponding locations, so that each sensor node is covered by at least one relay node, and that the set of relay nodes is connected. It Ensures each SN is covered by at least $1 \mathrm{RN}$ node. At least $1 \mathrm{RN}$ should be present within TR of $\mathrm{SN}$. This RN should be reachable to and from $\mathrm{CH}$. This ensures connected $\mathrm{SN}-\mathrm{RN}-\mathrm{CH}$ graph

(1) For each Cluster

(2) Input all SN in cluster in List, L

(3) While L!=NULL

(4) \{

(5) Select sensor node with largest distance from its $\mathrm{CH}$

(6) If(RN in transmission radius of sensor node ie SNRange)

(7) \{

(8) Ensure that $\mathrm{RN}$ can reach $\mathrm{CH}$

(9) $\}$

(10) Else

(11) \{

(12) Place $\mathrm{RN}$ between $\mathrm{CH}$ and $\mathrm{SN}$ such that $\mathrm{RN}$ is at distance SNRange from SN. The direction should be the line of sight of $\mathrm{SN}$ and $\mathrm{CH}$. Ensure that RN placed can reach $\mathrm{CH}$.

(13) Place more $\mathrm{RN}$ on line of sight of current $\mathrm{RN}$ and $\mathrm{CH}$

(14) $\}$

(15) Remove SN from List, L

(16) $\}$

-1 Cluster-wise run this algorithm to place Relay nodes inside cluster

-2 Insert all $\mathrm{SN}$ of cluster in a list so that we can verify that each $\mathrm{SN}$ can reach at least $1 \mathrm{RN}$.

-Repeat until all the sensor node have been treated. In the loop either the SN will have a relay node around it or it wont.

-4\&5. Select SN which is farthest from CH. This way Relay nodes placed will be minimum in number because the relay nodes will be placed in collinear with $\mathrm{SN}$ and $\mathrm{CH}$ and towards the $\mathrm{CH}$ at distance SNRange from $\mathrm{SN}$.

-6. Check if RN is found within the SNRange for selected $\mathrm{SN}$ in while loop

$-7,8,9$ : if $\mathrm{RN}$ is present ensure that this $\mathrm{RN}$ can send transmissions to $\mathrm{CH}$. That is, it is connected to $\mathrm{CH}$ via other relay nodes

-10,11,12: if RN is not found within transmission range of selected SN, place a relay node such that:

(1) It is at distance SNRange from selected SN 
(2) It is on same line in which co-ordinates of $\mathrm{SN}$ and $\mathrm{CH}$ lie

(3) It is placed in between $\mathrm{CH}$ and $\mathrm{SN}$

-13. If distance between $\mathrm{RN}$ and $\mathrm{CH}$ is more than RNRange place more $\mathrm{RN}$ between them such that

(1) It is at distance RNRange from newly placed RN

(2) It is on same line in which co-ordinates of $\mathrm{RN}$ and $\mathrm{CH}$ lie

(3) It is placed in between $\mathrm{CH}$ and $\mathrm{RN}$.

-14 . Remove SN from list

4.2.4.2 WITHIN CLUSTER: Ensure single node, double cover problem.

Given a set of locations of uniformly distributed sensor nodes $\mathrm{S}$, the communication range of sensor nodes SNRange, and the communication range of relay nodes RNRange, find the minimum number of relay nodes and their corresponding locations so that each sensor node is covered by at least two relay nodes, and that the network of relay nodes is 2-connected.

(1) For each cluster

(2) Input nodes $\mathrm{SN}$ in List, $\mathrm{L}$

(3) While L!=NULL

(4) \{

(5) Choose $\mathrm{SN}$ one by one

(6) If(atleast two $\mathrm{RN}$ are not present within transmission radius of $\mathrm{SN}$ in same cluster)

(7) \{

(8) Place $\mathrm{RN}$ in transmission area of $\mathrm{SN}$ such that mathematical constrains described in equation $\mathrm{A}$ is followed

(9) $\}$

(10) Remove SN from List, L

(11) \}

-1.For each cluster in field repeat this algorithm

-2.Input all the $\mathrm{SN}$ in List such that double cover for each can be verified

-3. While list is not null

$-4,5$ : Choose SN one by one

-6.Check if two Relay Nodes are present with the Transmission radius of selected $\mathrm{SN}$ in step 5

$-7,8$ : If less than 2 two relay nodes are present then we place 1 more relay node around SN. We decide to place only one because CRNC algorithm ensures that each $\mathrm{SN}$ has atleast one relay node around it. Here we dont check reachability because mathematical modelling uses this constraint that $\mathrm{RN}$ used by other RN within SNRange of any sensor node is used by the RN which is now placed using Fault Tolerance algorithm

-9. If two or more $\mathrm{RN}$ is placed around $\mathrm{SN}$ do nothing

-10. Remove SN from List as it satisfies Fault-Tolerance

\section{SIMULATION}

MATLAB is used for simulating the algorithm. MATLAB is a highperformance language for technical computing. It integrates computation, visualization, and programming in an easy-to-use environment where problems and solutions are expressed in familiar mathematical notation.

\subsection{Performance Analysis}

\subsubsection{Simulation Parameters}

—Field size (MM) : 100m100m

—Location of BS(Base Station):75m; dBS ; 100m

-Number of nodes, $\mathrm{N}: 100$ nodes

-Cluster-head $(\mathrm{CH})$ probability : 0.1

-Initial Energy of sensor node : 0.02J

—ETX and ERX (Eelec) : $50 \mathrm{~nJ} / \mathrm{bit}$

-Free space ( fs ): $10 \mathrm{pJ} / \mathrm{bit} / \mathrm{m} 2$

—Multipath fading (mp) : $0.0013 \mathrm{pJ} / \mathrm{bit} / \mathrm{m} 4$

— The energy for aggregation (EDA): $5 \mathrm{~nJ} / \mathrm{bit} / \mathrm{signal}$

4.1.2 RESIDUAL ENERGY COMPARISON GRAPHS. We simulated the Leach algorithm and our proposed algorithm in MATLAB and ploted various graphs comparing the Residue Energy when a large wireless network of nodes is Clustered using the LEACH algorithm and the proposed Clustering algorithm. Following graph shows the comparison of Residual energies when 50 nodes have been used in our WSN.

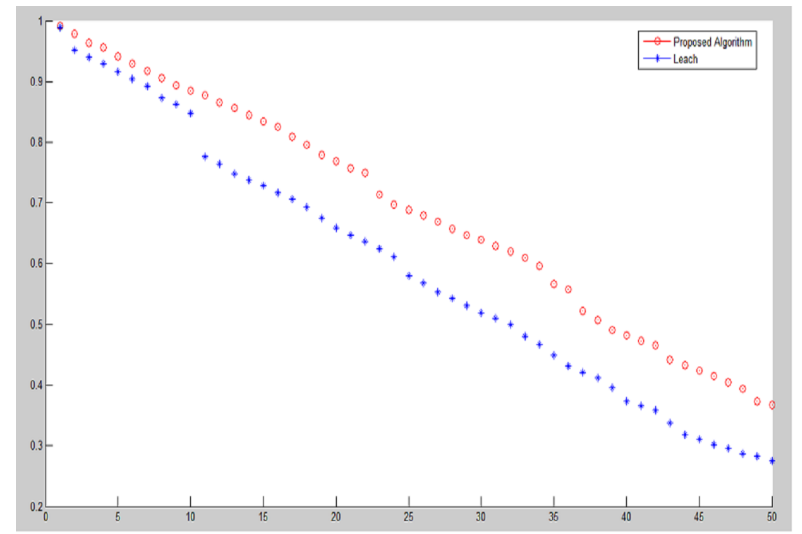

Fig. 1. Comparison of residual energies when 50 nodes have been used in our WSN.In the above scenario the Residual energy has increased from 0.6246 Joules in case of LEACH algorithm to 0.6600 Joules in case of the PROPOSED algorithm.

Figure 1 shows the Comparison of residual energies in case of LEACH algorithm and when the proposed algorithm was used.The number of nodes used is 50.T he Residual energy has increased from 0.6246 Joules in case of LEACH algorithm to 0.6600 Joules in case of the PROPOSED algorithm.

Figure 2 shows the Comparison of residual energies in case of LEACH algorithm and when the proposed algorithm was used.The number of nodes used in the WSN is 100 . The Residual energy has increased from 1.2266 Joules in case of LEACH algorithm to 1.3492 Joules in case of the PROPOSED algorithm. 


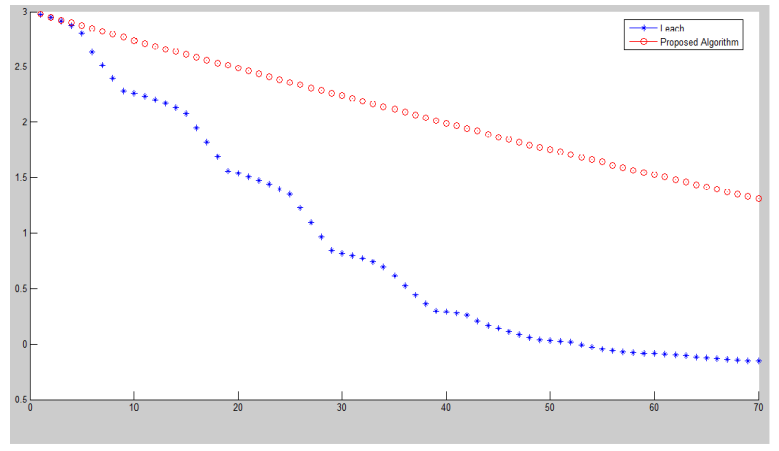

Fig. 2. Comparison of residual energies when 100 nodes have been used in our WSN.In the above scenario the Residual energy has increased from 1.2266 Joules in case of LEACH algorithm to 1.3492 Joules in case of the PROPOSED algorithm.

\section{RESULTS AND CONCLUSION}

The simulation of leach algorithm and the proposed algorithm has yield the following results in terms of Energy consumption and Network lifetime.

We have proposed a fine strategy for relay node placement in large scale, dense, non uniform sensor network. Five different cases have been discussed and their appropriate algorithms are proposed.The proposed algorithm suggested in 3.2.4 ensures connected relay node single cover situation within clusters. Single node, double cover problem is being handled efficiently by the algorithm described in section 3.2.4 (2). Placement of relay nodes is based on formed clusters.

In order to form the clusters, dominating set is used as a backbone in WSN. The algorithm used for constructed dominating set is a modified version of DSP-CDS[33]. Among the nodes belonging to the dominating set, cluster heads are chosen based on proposed algorithm 2. Finally, proposed Algorithm 3 is used during the formation of clusters.

Our approach has considered the combination of various factors that are relevant for efficient utilisation of power and thus minimising energy consumption in WSN. The algorithms are simulated on MATLAB R2007b and the results proved that the proposed algorithm increases the network lifetime and conserves the energy by $13.456 \%$ as compared against the LEACH algorithm.

\section{REFERENCES}

[1] I.F. Akyildiz, W. Su, Y. Sankarasubramaniam, E. Cayirci, Wireless sensor networks: a survey, Computer Networks Journal 38 (2002) 393- 422.

[2] Jian Tang*, Bin Hao, Arunabha Sen, Relay node placement in large scale wireless sensor networks, Computer Communications 29 (2006) 490501

[3] Y.T. Hou, Y. Shi, H.D. Sherali and S.F. Midkiff; Prolonging sensor network lifetime with energy provisioning and relay node placement; Secon05; pp. 295304.

[4] J. Bredin, E. Demaine, M. Hajiaghayi and D. Rus; Deploying sensor networks with guaranteed capacity and fault tolerance; Mobihoc05; pp. 309- 319.

[5] X. Cheng, D.Z. Du, L. Wang and B. Xu; Relay sensor placement in wireless sensor networks; ACM/Springer WINET07;
[6] A. Efrat, Sandor P. Fekete, P. Gaddehosur, J. Mitchell, V. Polishchuk and J. Suomela; Improved Approximation Algorithms for Relay Placement; ESA08; pp.356 367.

[7] B. Hao, J. Tang and G. Xue; Fault-tolerant relay node placement in wireless sensor networks: formulation and approximation; HPSR04; pp. 246-250.

[8] H. Liu, P.J. Wan and X.H. Jia; Fault-tolerant relay node placement in wireless sensor networks; LNCS; Vol. 3595(2005), pp. 230239.

[9] E. Lloyd and G. Xue; Relay node placement in wireless sensor networks; IEEE Transactions on Computers; Vol. 56(2007), pp. 134138.

[10] S. Misra, S. Hong, G. Xue and J. Tang; Constrained relay node placement in wireless sensor networks to meet connectivity and survivability requirements; Infocom08.

[11] ] J. Pan, Y.T. Hou, L. Cai, Y. Shi, S.X. Shen; Topology control for wireless sensor networks; Mobicom03, pp. 286299.

[12] A. Srinivas, G. Zussman, and E. Modiano; Mobile backbone networks Construction and maintenance, Mobihoc06, pp. 166177.

[13] K. Xu, H. Hassanein, G. Takahara and Q. Wang; Relay node deployment strategies in heterogeneous wireless sensor networks: multiple-hop communication case; Secon05, pp. 575585.

[14] W. Zhang, G. Xue, and S. Misra; Fault-tolerant relay node placement in wireless sensor networks: problems and algorithms; Infocom07; pp.16491657.

[15] A. Kashyap, S. Khuller and M. Shayman; Relay placement for higher order connectivity in wireless sensor networks; Infocom06.

[16] D. Estrin, R. Govindan, J. Heidemann, S. Kumar, Next century challenges: scalable coordination in sensor networks, ACM MobiCom99, Washingtion, USA, 1999, pp. 263270.

[17] G. Hoblos, M. Staroswiecki, A. Aitouche, Optimal design of fault tolerant sensor networks, IEEE International Conference on Control Applications, Anchorage, AK, September 2000, pp. 467472.

[18] D. Nadig, S.S. Iyengar, A new architecture for distributed sensor integration, Proceedings of IEEE Southeastcon93, Charlotte, NC, April 1993.

[19] C. Shen, C. Srisathapornphat, C. Jaikaeo, Sensor information networking architecture and applications, IEEE Personal Communications, August 2001, pp. 5259.

[20] E. Shih, S. Cho, N. Ickes, R. Min, A. Sinha, A. Wang, A. Chandrakasan, Physical layer driven protocol and algorithm design for energy-efficient wireless sensor networks, Proceedings of ACM MobiCom01, Rome, Italy, July 2001, pp. 272286

[21] S. Cho, A. Chandrakasan, Energy-efficient protocols for low duty cycle wireless microsensor, Proceedings of the 33rd Annual Hawaii International Conference on System Sciences, Maui, HI Vol. 2 (2000), p. 10

[22] N. Bulusu, D. Estrin, L. Girod, J. Heidemann, Scalable coordination for wireless sensor networks: self-configuring localization systems, International Symposium on Communication Theory and Applications (ISCTA 2001), Ambleside, UK, July 2001.

[23] C. Intanagonwiwat, R. Govindan, D. Estrin, Directed diffusion: a scalable and robust communication paradigm for 
sensor networks, Proceedings of the ACM Mobi- Com00, Boston, MA, 2000, pp. 5667.

[24] J.M. Kahn, R.H. Katz, K.S.J. Pister, Next century challenges: mobile networking for smart dust, Proceedings of the ACM MobiCom99, Washington, USA, 1999, pp. 271278.

[25] G.J. Pottie, W.J. Kaiser, Wireless integrated network sensors, Communications of the ACM 43 (5) (2000) 551558.

[26] A. Porret, T. Melly, C.C. Enz, E.A. Vittoz, A low-power low-voltage transceiver architecture suitable for wireless distributed sensors network, IEEE International Symposium on Circuits and Systems00, Geneva, Vol. 1, 2000, pp. 5659.

[27] Weiyi Zhang, Guoliang Xue and Satyajayant Misra, FaultTolerant Relay Node Placement in Wireless Sensor Networks: Problems and Algorithms, 2007 IEEE

[28] Y. Thomas Hou, Senior Member, IEEE, Yi Shi, Student Member, IEEE,Hanif D. Sherali, and Scott F. Midkiff, Senior Member, IEEE, On Energy Provisioning and Relay Node Placement for Wireless Sensor Networks, IEEE TRANSACTIONS ON WIRELESS COMMUNICATIONS, VOL. 4, NO. 5, SEPTEMBER 2005

[29] K. Lu G. Liu R. Mao Y. Feng, Relay node placement based on balancing power consumption in wireless sensor networks, The Institution of Engineering and Technology 2011

[30] Hai Liu, Peng-Jun Wan, and Xiaohua Jia, Fault-Tolerant Relay Node Placement in Wireless Sensor Networks, SpringerVerlag Berlin Heidelberg 2005

[31] Santhosh Pandey ,Shaoqiang Dong ,Prathima Agrawal ,Krishna M. Sivalingam, On Performance of Node Placement Approaches for Hierarchical Heterogeneous Sensor Networks, Springer Science + Business Media, LLC 2008

[32] Dejun Yang, Satyajayant Misra, Xi Fang, Guoliang Xue, and Junshan Zhang, Two-Tiered Constrained Relay Node Placement in Wireless Sensor Networks: Efficient Approximations,2010 IEEE

[33] R. Agrawal, and R. Srikant, Fast Algorithms for Mining Association Rules, Proceedings of the 20th VLDB, pp. 487499, 1994.

[34] S.K. Chong, S. Krishnaswamy, S.W. Loke, and Mohamed Gaber, Using Association Rules for Energy Conservation in Wireless Sensor Networks, Proceedings of the 23rd ACM Symposium on Applied Computing, Brazil, 2008.

[35] A. Deshpande, C. Guestrin, and S.R. Madden, Using Probabilistic Models for Data Management in Acquisitional Environments, Proceedings of the 2005 CIDR Conference, 2005.

[36] W. Heinzelman, A. Chandrakasan, and H. Balakrishnan. An Application-Specific Protocol Architecture for Wireless Microsensor Networks, IEEE Transactions on Wireless Communications, Vol. 1, pp. 600-670, 2002.

[37] W. Hu, V.N. Tran, N. Bulusu, C.T. Chou, S. Jha, and A. Taylor. The Design and Evaluation of a Hybrid Sensor Network for Canetoad Monitoring, Proceedings of Information Processing in Sensor Networks (IPSN 2005/SPOTS 2005), Los Angeles, 2005.

[38] P. Levis, N. Lee, M. Welsh, and D. Culler, TOSSIM: Accurate and Scalable Simulation of Entire TinyOS Applications, SenSys 2003, 2003.

[39] K.K. Loo, I. Tong, B. Kao, and D. Cheung, Online Algorithms for Mining Inter-Stream Associations From Large Sensor Networks, PAKDD, pp. 143-149, 2005.
[40] R. Marin-Perianu, M. Marin-Perianu, and P. Havinga, Movementbased Group Awareness with Wireless Sensor Networks, Proceedings of Pervasive 2007, 2007.

[41] V.S. Anitha, M.P. Sebastian, A Connected Dominating Setbased Weighted Clustering Algorithm for Wireless Sensor Networks, IEEE 2010

[42] L. Jia, R. Rajaraman, and T. Suel, An efficient distributed algorithm for constructing small dominating sets, Distrib. Comput., vol. 15, no. 4, pp. 193205, 2002.

[43] Julia Albath, Mayur Thakur, Sanjay Madria, Energy Constrained Dominating Set for Clustering in Wireless Sensor Networks, 24th IEEE International Conference on Advanced Information Networking and Applications, 2-5, 2010

[44] Bolian Yin, Hongchi Shi, Yi Shang, An efficient algorithm for constructing a connected dominating set in mobile ad hoc networks, J. Parallel Distributed Computing 71(2011) 27-39

[45] Do-hyun Nam, hong-ki min, An Efficient Ad-Hoc Routing Using aHybrid Clustering Method in a Wireless Sensor Network, Wirelessand Mobile Computing, Networking and Communications, pp. 60-60, Oct 2007.

[46] A.A. Abbasi and M. Younis, A survey on clustering algorithms for wireless sensor networks, Computer Communications, 30, 28262841, 2007.

[47] Wendi B Heinzelman, Anantha P. Chandrashekhan, Hari Balakrishan, An application specific protocol architecture for wireless Senesor Network, IEEE 2002

[48] Ma Chaw Mon Thein, Thandar Thein , An energy efficient Cluster Head Selection for Wireless Sensor network, 2010 Int. Conf. on Intelligent systems, Modelling and simulation.

[49] 0. Younis, et. al., HEED: A Hybrid, Energy-Efficient, Distributed Clustering Approach for AdHoc Sensor Networks, IEEE Transactions on Mobile Computing, 3(4):660-669, 2004.

[50] S. Lindsey, et. al. ,PEG ASIS: Power-Efficient Gathering in Sensor Information Systems, IEEE Aerospace Conference Proceedings, Vol. 3, 9-16 gust, pp. 102-114, 2002.

[51] Shujuan Jin, Keqiu Li, LBCS: A Load Balanced Clustering Scheme in Wireless Sensor Networks, 2009 Third International Conference on Multimedia and Ubiquitous Engineering, IEEE

[52] Suan Khai Chong, Mohamed Medhat Gaber, A rule Learning approach to energy efficient clustering in wireless sensor network , 2008 IEEE 